\title{
Multiobjective Optimization of IPM synchronous motor using Response Surface Methodology and filtered Monte Carlo approach
}

\author{
R. Seifert, R. Bargalló Perpiñà
}

\begin{abstract}
Permanent Magnet Synchronous Motors offer high efficiency and power density besides low assembly effort and hence have been established in a wide market over the past years. Especially buried magnets enable a superior field weakening ability but require an exceptional design effort. Costly Finite Elements computations are inevitable for consideration of the occurring non-linearity and non-trivial magnet shapes. The Response Surface Methodology can reduce the number of FE runs significantly by introducing an acceptably exact second order regression model based on a few carefully chosen design samples. Instead of commonly used, but time-consuming Evolutionary Strategy methods, the Monte Carlo approach is applied for optimization. Using simple filter algorithms, distinctive Pareto frontiers can be determined quickly and related to their causative motor designs.
\end{abstract}

Index Terms: FE methods, IPM, Optimization and Pareto frontiers, Response Surface Methodology.

\section{I.INTRODUCTION}

NTERIOR permanent magnet synchronous motors (IPMSM) became established in a wide field of applications, especially in case compact designs with high power capability are required. Due to the absence of rotor and commutator losses they are characterized by an excellent efficiency. The additional reluctance torque provides a high torque over a wide speed range. Most likely inverter driven they provide a superior controllability and a high power density, when operated at the optimum torque-to-ampere trajectory. However despite all advantages high magnet costs hamper the entering into the mass market of electrical excited machines. In spite of the exploding world-market prices for rare earth materials and magnets [1], their benefits over ferrite magnets in terms of size, weight and performance are so conclusive, that they are still dominating the market. In contrary to surface mounted magnets, which are often bent and skewed and adapted for certain rotor designs, interior magnets get along with simple shapes, which even can be used in different layouts of different sizes. This way the wastage and therefore production costs can be minimized. The excitation through buried magnets enables a rugged rotor construction which allows high speeds and requires low maintenance, as the magnets are physically protected and fixed without the need of bandages at high speeds. This in turn allows small air gaps and a further increased efficiency. Technically, the introduced steel poles alter the magnetic circuit, weak the d-axis path and hence capacitate the complete cancellation of the excitation field. On the other hand they strengthen the q-axis

Robert Seifert is with Elektrotechnisches Institut Dresden University of Technology Email: Robert.Seifert@mailbox.tu-dresden.de

Ramon Bargalló Perpiñà is with Electrical Engineering Department. Polytechnic University of Catalonia. EUETIB, C/Urgell, 187, 08036 Barcelona, Spain (e-mail: ramon.bargallo@upc.edu) due to an dominating quadrature axis inductance, are leading to a high saliency of the magnetic circuit and introducing an additional reluctance torque component, which makes IPMSM superior for field weakening demanding applications, like pump/fan drives or machine tool servo drives. Because low flux linkages at high speeds not automatically cause decreased torques at low speeds, as the reluctance torque is independent of the permanent magnet flux. Cogging torque can be minimized by a deliberate rotor design, even without the otherwise commonly used skewing, which is only hardly feasible for IPMSM. Air barriers to prevent magnetic short circuits and the occasionally wide spread steel poles cause a non-trivial flux distribution regarding the rotor, which complicates the design procedure considerably. Analytic models relating rotor geometry and air gap field fail to describe satisfactory basic correlations. The use of the FE analysis is inevitable to determine the fundamental waves in the air gap, whose evaluation then can be applied on analytic models. The objective of this paper is to introduce the combined use of Finite Element Analysis, Response Surface Methodology and Monte Carlo Method for an effective reduction of computation time.

\section{ANALYTIC MODEL OF THE IPMSM}

The first attempts of analyzing the field weakening abilities using the linear lossless model of IPMSM date back to the 80 's. Inverter voltage and stator currents are related to torque and power output considering $\mathrm{d}$ - and q-axis inductance as constant motor parameters. The influence of the saturation of the magnetic circuit is neglected as well as stator and iron losses. However the model is sufficient to describe the drive characteristics and power capability of the motor over the entire operating range and allows a comparison of different motor designs, but is not capable of determining realistic motor characteristics and is therefore disqualified for optimization purposes. The development of the analytic model is highly shortened and just relevant correlations are shown. For an extensive derivation I refer to [3] for further reading, a comparison of analytic model and FE-analysis is shown in [4].

\section{A. Analytic model}

Based on the magnetic $\lambda_{\mathrm{m}}$ and the $\mathrm{d} / \mathrm{q}$ - inductances as well as currents the torque can be calculated by

$$
T=T_{a l}+T_{r e l}=\frac{3}{2} \cdot p \cdot\left(\lambda_{m} \cdot I_{q}\right)+\frac{3}{2} \cdot p \cdot\left(L_{d}-L_{q}\right) \cdot I_{d} \cdot I_{q}
$$

In this equation becomes apparent why IPMSM are often denoted as a hybrid combination of the conventional synchronous-reluctance SM and surface mounted PMSM. [5] The first term is called field-alignment torque $T_{a l}$, the second one reluctance torque $T_{\text {rel }}$, which is highly dependent 
on the saliency ratio $\xi=\mathrm{L}_{\mathrm{q}} / \mathrm{L}_{\mathrm{d}}$. Under saturation the flux linkages, especially the q-axis flux, are not proportional to the stator currents. The inductances decrease significantly in the most saturated driving regions for maximum q-axis current $\left(\beta=90^{\circ}\right)$ Rather its use as parameter for torque optimization should be avoided completely(In the experiments the q-axis inductance was reduced about $24 \%$ for maximum q-axis current, equally the saliency ratio as the direct axis was just slightly influenced. Further analysis regarding saturation effects was made by Soong and Miller [6]) Instead all calculations are carried out using the flux linkages directly, computed by the FE-analysis. To contain the number of numeric computations the stator current is fixed at the rated current circle and therefore the variable parameters are reduced to a single one, the electric angle $\beta$.

$$
I_{d}=I_{r} \cdot \cos \beta ; I_{q}=I_{r} \cdot \sin \beta
$$

Consequently the flux linkages for rated current $I_{r}$, and therefore the according voltages, are just functions of $\beta$, while it is sufficient to observe them in the range of $\beta=\left[90^{\circ}\right.$; $180^{\circ}$ ]

$$
\begin{aligned}
& \lambda_{d}(\beta)=\lambda_{m}+\lambda_{s d}\left(I_{d}\right) ; \lambda_{q}(\beta)=\lambda_{s q}\left(I_{q}\right) \\
& V_{d}=-\omega \cdot \lambda_{d}\left(I_{q}\right) ; V_{q}=\omega \cdot \lambda_{s d}\left(I_{d}\right)
\end{aligned}
$$

For the torque follows

$$
T=\frac{3}{2} \cdot p \cdot I_{r} \cdot\left(\lambda_{d}(\beta) \cdot \sin (\beta)+\lambda_{q}(\beta) \cdot \cos (\beta)\right)
$$

\section{B. Drive operation limits}

Besides mechanical restrictions, the main limitations of the drive regions are the maximum output voltage of the inverter and the maximum acceptable stator current under thermal aspects.

$$
I_{d}^{2}+I_{q}^{2} \leq I_{r}^{2} ; V_{d}^{2}+V_{q}^{2} \leq V_{\max }^{2}
$$

Defining an ellipse

$$
\left(\lambda_{m}+L_{d} \cdot I_{d}\right)^{2}+\left(L_{q} \cdot I_{q}\right)^{2} \leq\left(V_{\max } / \omega\right)^{2}
$$

Which has its center in point [CVL; 0] (Fig. 1), with

$$
C V L=-\frac{\lambda_{m}}{L_{d}}=\frac{\lambda_{m}}{\lambda_{d s}} \cdot I_{r}\left(\beta=180^{\circ}\right)=-\frac{\lambda_{m}}{\lambda_{d s}} \cdot I_{r}
$$

The ratio $\lambda_{m} / \lambda_{d s}$, respectively the difference $\lambda_{m}-\lambda_{d s}$ is a very suitable criterion to optimize the constant power speed range (CPSR) as it directly expresses the field weakening ability. It is described by the ratio $\Omega_{2} / \Omega_{1}$ of maximum speed $\Omega_{2}$ to minimum speed $\Omega_{1}=\Omega_{\mathrm{r}}$ at which rated power can be achieved. Furthermore hyperbolas of constant torque can be defined using eq. 1 :

$$
I_{q}=\frac{2 \cdot T}{3 \cdot p \cdot L_{d}} \cdot\left(\frac{\lambda_{m}}{L_{d}}+(1-\xi) \cdot I_{d}\right)^{-1}
$$

Practically every specific value of torque (below $T_{\max }$ ) can be attained by a indefinite number of current vectors. To maximize efficiency Jahns [5] introduced a current trajectory for an optimal 'maximum torque per stator current ampere' characteristic, in which the current vector follows the normal vectors of the torque hyperbolas, in other words it is to choose the point on every torque hyperbola as close as possible to the origin of the $\mathrm{d}$ /q-plane (Mode 0 in Fig. 1). By derivation of eq. 1 and transposition, the current angle $\beta_{\text {Tmax }}$ for maximum torque can be determined [7]. For speeds below base speed $\Omega_{\mathrm{b}}$ the voltage demand, which is necessary to maintain the maximum torque, reaches the limit of the inverter (Point A in Fig. 1). So torque and power are just limited by the current (Mode I). Above $b$ the current vector is following the current limit circle (Mode II), where voltage limit ellipses and constant torque hyperbolas have intersections. This leads us to the fact, that a classification in two machine types is necessary.

Class I - Finite speed IPMSM: Machines of class I are usually excited by rare earth magnets and have a high saliency. Even the maximum negative d-axis current is not able to completely cancel out the magnetic flux, which entails a finite maximum speed at point $C$. The current vector remains at the rated current circle and the machine is kept in Mode II for optimal field weakening performance.

Class II - Infinite speed IPMSM These machines are excited by weaker magnets, e.g. ferrite, and/or have a relatively low saliency. Moving on the current limit circle the total $\mathrm{d}$-axis flux would become negative at a certain angle $\beta$ (point $\mathrm{B}$ ) and decrease the torque rapidly. For an optimum torque/current trajectory (Mode III) the d-axis current needs to be reduced in such away that constant torque hyperbolas and voltage limit ellipses osculate tangential. As the latter is centered inside the current circle, the speed can theoretically rise to infinite.

This differentiation was first made by Morimoto [7] and extended by Soong and Miller [8] by classifying and comparing these two types with other classes of brushless synchronous AC motor drives. Together with Jolly [4] they all refer to the optimal field weakening design at the changeover of these two classes. [8] The basic concept is to reduce $\mathrm{q}$-axis as well as d-axis flux to zero to achieve the maximum field weakening ability. For the q-axis this is generally the case for $\beta=180_{\text {a }}$ as $\mathrm{I}_{\mathrm{q}}$ is zero. So the points of neutralization of d-axis stator flux $\lambda_{\mathrm{sd}}$ and magnetic flux $\lambda_{\mathrm{m}}$ has to coincide with this electric angle. In this case the speed reaches its maximum or infinite for total neutralization.

$$
\Omega_{\max }=\frac{V_{\max }}{p \cdot \sqrt{\lambda_{d}^{2}(\beta=\pi)+\lambda_{q}^{2}(\beta=\pi)}}
$$

For infinite speed IPMSM, this can be obtained by regulating the stator current. But in this manner the utilization of the machine is below capacity as the thermal limits allow higher currents. Hence the neutralization is desired for rated current. Thus the voltage limit ellipse is centered at the rated current circle. The essential design objectives for optimal field weakening performance, first stated by Schiferl [9], are:

$$
\left.\frac{\lambda_{m}}{\lambda_{s}}\right|_{I_{d}=-I_{r}, I_{q}=0}=1
$$




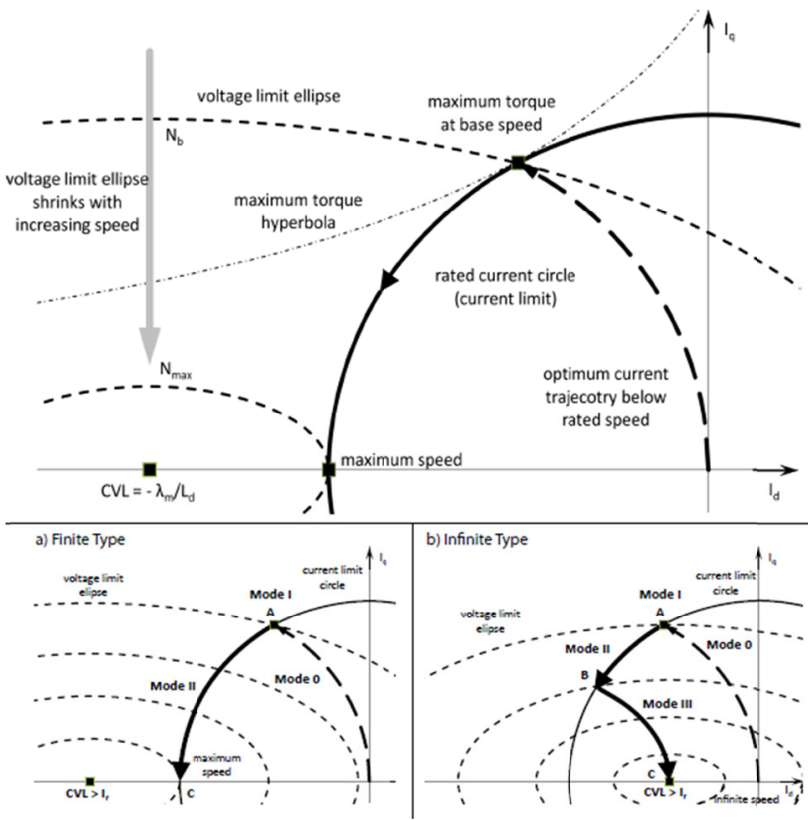

Fig. 1: Drive operation limits of IPMSM, for a) finite type and b) infinite type [7] [8]

\section{RESPONSE SURFACE METHODOLOGY}

The Response Surface Methodology (RSM) was first introduced by Box and Wilson [10] in 1951. RSM is efficacious when the process has no analytical expression to describe it or when the analytical expression is too complex and when there are some indeterminate factors not modeled. RSM creates an empirical model that relates the process response to well-known input parameters. Its application to electromagnetic problems started in the 90's [11] and other applications are founded on Jabbar [12] and Jolly [4] in the last decade. The purpose is to achieve a set of design variables $\gamma$ in which the response $\eta$ reach a maximum or a minimum within an experimental region $\mathcal{R}$, which is defined by practical limitations like geometry, mechanical restrictions or saturation. The $k$-independent design variables are normalized to equally bounded factors $x_{1} ; x_{2} ; \ldots, x_{k}$ and these need to be capable of an exact measurement. In order to determine the response $\hat{y}$, the regression method of least squares is applied for multiple order polynomials, fitting the sure values $y$ ( $n$ design samples computed by FE-analysis). To minimize the computing time it is desired to keep $n$ as small as possible, which is dependent on the polynomial order and the number of factors $k$. The Design of Experiments (DoE) is a tool used for determining the optimal test points for RSM. RSM cannot substitute measurements and additional FE computations in the experimental region around the optimized response, but reduce them to a feasible number.

\section{A. Procedure}

Every process is characterized by a true response such as the actual torque for a motor, which can be measured and hence underlies a measurement error $\varepsilon_{\mathrm{m}}$. It is selfexplanatory that there are no prototypes available for every design sample to verify the FEM computations. For this reason a single computation result itself is assumed to be an adequate image of a real machine and defined as the true response $\eta$. However the evaluation of the FE-analysis is not always trivial and underlies an error $\varepsilon_{\text {eva }}$. Considering this, the so-called sure value or mean response $y$ is described as:

$$
y=\eta+\varepsilon_{\text {eva }}=f\left(x_{1}, x_{2}, \ldots . x_{i}\right)+\varepsilon_{\text {eva }}
$$

The evaluation of the error is random and assumed to have zero mean value and thereby $y$ is conditioned to be an adequate representation of the true response $\eta$. For relating the response to the input variables, the approximation function $f(1)$ needs to be attained. This is accomplished by means of a low-degree polynomial represented by a matrix of arguments $X$ and a matrix of coefficients $\beta$ :

$$
y=X \cdot \beta+\varepsilon_{\text {eva }}
$$

As an example, for the second-degree model $(d=2)$ we can write:

$$
y=\beta_{0}+\sum \beta_{i} \cdot x_{i}+\sum \sum \beta_{i j} \cdot x_{i} \cdot x_{j}
$$

In general the number of terms and coefficients of a $k$ variables and $d$-degree polynomials can be determined as:

$$
c=\left(\begin{array}{c}
k+d \\
d
\end{array}\right)=\frac{(k+d) !}{k ! \cdot d !}
$$

The design samples are represented by a matrix $D=\left[D_{1}\right.$, $\mathrm{D}_{2}, \ldots \mathrm{D}_{\mathrm{n}}$ ] containing the different geometries, for which the response $y$ has been computed previously by the FEanalysis. This leads us to the following over-determined linear system of equations:

$$
Y=\left(\begin{array}{c}
y_{1} \\
y_{2} \\
\cdots \\
y_{n}
\end{array}\right)=\left(\begin{array}{c}
X_{1} \\
X_{2} \\
\cdots \\
X_{n}
\end{array}\right) \cdot \beta+\epsilon=Z \cdot \beta+\epsilon
$$

The coefficient vector $\beta$ is determined under the approach of linear least squares [13]. Its so-called ordinary leastsquares estimator $\beta$ and henceforth the predicted response $\hat{y}$ for every single point in the experimental region $\mathcal{R}$ can be obtained by:

$$
\left.\begin{array}{c}
\hat{\beta}=\left(Z \cdot Z^{T}\right)^{-1} \cdot Z^{T} \cdot Y \\
\hat{y}=\hat{\beta} \cdot X
\end{array}\right\}
$$

TABLE I

Statistical attributes

\begin{tabular}{|l|c|}
\hline \multicolumn{1}{|c|}{ Name } & Equation \\
\hline Mean value of all mean responses & $\bar{y}=\sum y_{i} / n$ \\
\hline Average relative error & $\overline{\Delta y / y}=\frac{1}{n} \cdot \sum\left|\frac{y_{i}-\widehat{y}_{i}}{y_{i}}\right|$ \\
\hline Maximum relative error & $\max |\overline{\Delta y / y}|=\max \left|\frac{y_{i}-\widehat{y}_{i}}{y_{i}}\right|$ \\
\hline $\begin{array}{l}\text { Total sum of squares (variance of } \\
\text { samples) }\end{array}$ & $S S T=\sum\left(y_{i}-\bar{y}\right)^{2}$ \\
\hline $\begin{array}{l}\text { Regression sum of squares (var. of } \\
\text { responses) }\end{array}$ & $S S E=\sum\left(\hat{y}_{i}-\bar{y}\right)^{2}$ \\
\hline $\begin{array}{l}\text { Residual sum of squares (var. of } \\
\text { errors) }\end{array}$ & $R^{2}=1-\frac{S S E}{S S T}$ \\
\hline $\begin{array}{l}\text { Coefficient of determination } \\
\text { cof } \\
\text { determination }\end{array}$ & $\tilde{R}^{2}=1-\left(1-R^{2}\right) \cdot \frac{n-1}{n-c-1}$ \\
\hline \multicolumn{2}{|l|}{ coefficient $\quad$ of } \\
\hline
\end{tabular}




\section{B. Error estimation}

There are various statistical attributes, which can evaluate the accuracy of a regression model (Table I). In case the regression model just involves interpolations and no extrapolations, the summation of SSE and SSR equals the total sum of squares SST. For instance in case the whole experimental region $\mathfrak{R}$ lies inside of the space framed by the Full Factorial Design. The explained coefficient of determination $\mathrm{R}^{2}$ is commonly used for the rating of regression models ( $1=$ ideal regression), even though it is not utterly correct for all cases. Preferable is the unexplained coefficient $\mathrm{R}^{2}$, which also involves the number of sampling points and the degree of the polynomial represented by its number of terms c (eq. 15)

\section{Introduction of different RSM designs}

Generally it is desired to minimize the number of samples, which need to exceed the number of coefficients $c$ of the underlying multi-order tri-variate polynomials. Consequently for second-order polynomials 10 coefficients are required, which can be provided by all of the following introduced RSM designs, but just the Full Factorial Design is applicable to third-order polynomials involving 20 terms.

1) Full Factorial Design (FFD): also called 3k Factorial Design is commonly used due to its high accuracy, though it is the most time-consuming design considering $3^{\mathrm{k}}=27$ design samples. Every design variable can take a value [-1 0 1]. It has the characteristic property of orthogonality. A design is orthogonal if the correlation matrix ZZ' results in a diagonal matrix. For this reason the significance of unknown parameters can be evaluated easier, as both $\beta$ and $\varepsilon$ (assuming normal distribution) are statistically independent.

2) Box Behnken Design (BBD): developed in 1960 [14], is a simplification of the FFD and omits the vertex and face center points and therefore requires only 13 computations. On closer inspection it becomes apparent, that every level of every design variable is represented by an equal number of four points, the origin excluded. In some scientific problems it is desired to analyze these layers, called blocks, independently under comparable conditions. The response variance within a single block is equal and uncorrelated to nearby blocks, which is called orthogonal blocking.

3) Central Composite Design (CCD): is referred as one of the most popular ones [13] and was introduced by Box and Wilson in 1951 [10]. It emanates from the usual approach of starting with a first-order polynomial model, considering just the vertexes of a simple cube, to identify initial tendencies. Subsequently further points are added to improve the model. Besides the origin these are usually face centered points (Face Centered Design) or star points, which then should enable the rotatability of the design. That means the prediction variance $\operatorname{Var}(\mathrm{y})$ is constant for all responses equidistant from the origin, a property which becomes useful for spherical problems.

\section{Design of experiments}

Within the design of experiments (DoE) is declared how the experimental region $\mathfrak{R}$ is embedded in the chosen RSM design. Certain levels $\left[\mathrm{x}_{1} ; \mathrm{x}_{2} ; \ldots ; \mathrm{x}_{\mathrm{k}}\right]$ are defined by physical boundaries, which should be identified correctly to exclude inadequate responses and keep $\mathfrak{R}$ as small as possible. The parameter levels are generally coded as:

$$
x_{\gamma}=\alpha_{S P} \cdot \frac{2 \cdot \gamma-\left(\gamma_{\max }+\gamma_{\min }\right)}{\left.\gamma_{\max }-\gamma_{\min }\right)}
$$

in which $\gamma$ is the physical design variable and $\alpha_{\mathrm{SP}}$ the star point factor, in case the CCD is used. Indeed $\mathfrak{R}$ is considerably larger than the space actually covered by FFD and BBD, so outer responses meed to be extrapolated which can cause a loss of accuracy. This difficulty appears since this paper intends to compare different RSM designs while introducing the optimization process. If a motor design is supposed to be optimized exclusively by FFD or BBD, their design matrix $\mathrm{D}$ is adapted fittingly to $\mathfrak{R}$ (compare TABLE $\mathrm{IIb}$ ). For the optimization problem discussed in this paper, the design variables $\gamma$ are the magnet position $\delta \mathrm{R}$ (distance from shaft) and the outer arc length of the air barriers, defined by the angles $\alpha_{1}$ and $\alpha_{2}$ (Fig. 5).

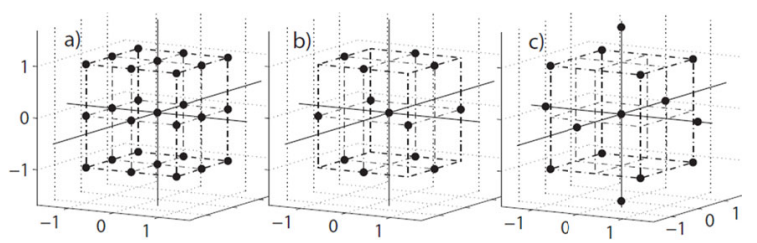

Fig. 2: Full Factorial Design (FFD), Central Composite Design (CCD) and Box Behnken Design.

\section{E. Comparison of RSM designs}

1) General comparison: For the most optimization problems the maximum torque $\mathrm{T}$ is the main objective. It is decisive whether or not a motor can be used for certain applications. Furthermore it highly influences the efficiency and power output. Within $\mathfrak{R}$ it varies only slightly, because the probed geometry is not as crucial as electro-magnetic factors like the remanent field strength of the permanent magnets or the actual applied current. Hence good regression results by the use of RSM can be expected. Except the firstorder $\mathrm{FFD}_{1}$ all RSM designs yield to satisfactory regression results and even the former would be suitable for the prediction of tendencies. Although CCD and BBD stand back behind the comparable $\mathrm{FFD}_{2}$ design, their relative errors are virtually identical. The use of higher polynomials, if possible, can further improve accuracy. For a proper DoE or reduced $\mathrm{R}, \mathrm{FFD}_{2}$ and $\mathrm{BBD}$ outperform or at least match $\mathrm{CCD}$ in terms of accuracy and $\mathrm{FFD}_{3}$ minimizes its errors to almost zero (TABLE IIb). Another often desired motor design objective is the field weakening ability, characterized by the maximum speed $\mathrm{N}_{\max }$ and the constant power speed range CPSR. In TABLE IId,e becomes apparent, that these variables are not suitable as optimization objectives at all. So the d-axis flux $\lambda_{d}$ for $\beta=\pi$ (henceforth denoted as $\lambda_{\pi}$ ) is used as it directly quantifies the field weakening. The accuracy for the regression of $\lambda_{\pi}$ is lower than for $T_{\max }$ (TABLE IIc), but much more important as a certain value $\lambda_{\pi}$ $=0$ needs to be achieved for optimal performance. Manual optimization by trial-and-error in the predicted optimum region is inevitable. Due to the small absolute values of $\lambda_{d}$ around zero, the relative errors increase significantly. For this reason the ratio of average absolute error to the average 
response value $\Delta y / y$ is determined, to show that the absolute error still remain very small and allow a satisfactory regression. The vertex point $\mathrm{D}_{22}$ is excluded in the $\mathrm{BBD}$, which is the most influential design sample for the optimization of the field weakening region. For this purpose BBD is discarded.

TABLE II

Errors and coefficients of determination

\begin{tabular}{|c|c|c|c|c|c|}
\hline & $\mathrm{FFD}_{1}$ & $\mathrm{FFD}_{2}$ & $\mathrm{FFD}_{3}$ & $\mathrm{CCD}$ & BBD \\
\hline \multicolumn{6}{|c|}{ a) For $T_{\max }$} \\
\hline$R^{2}$ & 0.59 & 0.96 & 0.98 & 0.96 & 0.96 \\
\hline$\tilde{R}^{2}$ & 0.52 & 0.93 & 0.90 & 0.87 & 0.74 \\
\hline$\overline{\overline{\Delta y / y}}$ & 2.54 & 0.65 & 0.36 & 0.66 & 0.67 \\
\hline $\max |\overline{\Delta y / y}|$ & 6.14 & 3.82 & 3.14 & 2.07 & 3.58 \\
\hline$\overline{\Delta y} / \bar{y}$ & 2.51 & 0.65 & 0.35 & 0.66 & 0.66 \\
\hline \multicolumn{6}{|c|}{ b) $\quad$ For $T_{\max }$ and reduced $\mathrm{R}$} \\
\hline$R^{2}$ & 0.56 & 0.98 & 1 & 0.96 & 0.98 \\
\hline$\tilde{R}^{2}$ & 0.48 & 0.97 & 0.99 & 0.87 & 0.87 \\
\hline$\overline{\Delta y / y}$ & 2.56 & 0.50 & 0.16 & 0.62 & 0.51 \\
\hline $\max \mid \overline{\Delta y / y}$ & 2.53 & 0.5 & 0.16 & 0.63 & 0.51 \\
\hline \multicolumn{6}{|l|}{ c) For $\lambda_{\pi}$} \\
\hline$R^{2}$ & 0.9 & 0.98 & 0.98 & 0.98 & 0.98 \\
\hline$\tilde{R}^{2}$ & 0.88 & 0.97 & 0.92 & 0.94 & 0.87 \\
\hline$\overline{\overline{\Delta y / y}}$ & 53.87 & 14.24 & 4.80 & 5.42 & 18.38 \\
\hline$\overline{\max |\overline{\Delta y / y}|}$ & 1430.74 & 286.52 & 49.76 & 24.69 & 424.25 \\
\hline$\overline{\Delta y} / \bar{y}$ & 9.02 & 3.98 & 2.41 & 3.92 & 4.18 \\
\hline \multicolumn{6}{|c|}{ d) For CPSR } \\
\hline$R^{2}$ & 0.17 & 0.47 & 0.73 & 0.35 & 0.08 \\
\hline$\tilde{R}^{2}$ & 0.02 & 0.13 & -0.16 & -1.26 & -4.50 \\
\hline$\overline{\Delta y / y}$ & 185.14 & 180.51 & 186.74 & 254.24 & 16.41 \\
\hline $\max |\overline{\Delta y / y}|$ & 545.61 & 689.60 & 695.92 & 884.81 & 91.19 \\
\hline \multicolumn{6}{|l|}{ Derived from $\lambda_{\pi}$} \\
\hline$\overline{\Delta y / y}$ & 12.20 & 8.09 & 7.57 & 7.89 & 8.45 \\
\hline $\max \mid \overline{\Delta y / y}$ & 90.6 & 43.43 & 69.08 & 33.84 & 65.86 \\
\hline \multicolumn{6}{|l|}{ e) For $N_{\max }$} \\
\hline$R^{2}$ & 0.17 & 0.46 & 0.73 & 0.35 & 0.07 \\
\hline$\tilde{R}^{2}$ & 0.01 & 0.12 & -0.17 & -1.28 & -4.56 \\
\hline$\overline{\Delta y / y}$ & 265.10 & 255.60 & 277.35 & 363.57 & 20.65 \\
\hline$\overline{\max |\overline{\Delta y / y}|}$ & 863.27 & 1096.4 & 1140.5 & 1409.3 & 92.57 \\
\hline \multicolumn{6}{|l|}{ Derived from $\lambda_{\pi}$} \\
\hline$\overline{\overline{\Delta y / y}}$ & 11.61 & 6.29 & 5.30 & 5.52 & 6.95 \\
\hline $\max |\overline{\Delta y / y}|$ & 92.45 & 46.12 & 78.70 & 32.80 & 69.00 \\
\hline
\end{tabular}

2) Application on the non-linear model: The field weakening ability can be determined by a single FE computation for $I_{q}=0$ and $I_{d}=I_{r}$, but for maximum torque the electric angle _ is not constant within $\mathfrak{R}$. Hence it is necessary to evaluate the entire second quadrant of the rated current circle, considering all saturation effects.

3) Selection of suitable optimization objectives: It was claimed before, that $\mathrm{N}_{\max }$ and CPSR are no suitable optimization objectives, as they are able to reach an infinite value. This behavior cannot be handled by the regression models. The occurring errors are unacceptable. Rather the flux linkage $\lambda$ is used as optimization objective as it is the actual initiator of the hyperbolic behavior The maximum speed can therefore be estimated indirectly using eq. 10, reducing the errors to satisfactory levels and enable rough predictions, as small estimation errors of $\lambda$ are still increased substantial by the hyperbolic function:

$$
\frac{N_{\max }}{r p m}=\frac{V_{\max }}{2 \cdot p \cdot \pi \cdot \sqrt{\lambda_{\pi}^{2}}} \cdot \frac{W b}{V}=1.0982 \cdot 10^{-4} \cdot\left(\frac{\sqrt{\lambda_{\pi}^{2}}}{W b}\right)^{-1}
$$

For the regression of CPSR no trivial equation is available and a $\mathrm{x}^{\mathrm{a}}$ polynomial is used, fitting $\mathrm{Y}_{\lambda}$ with an accuracy of $\mathrm{R}^{2}=0.9999$.

$$
C P S R=0.285 \cdot\left(\frac{\sqrt{\lambda_{\pi}^{2}}}{W b}\right)^{-0.913}
$$
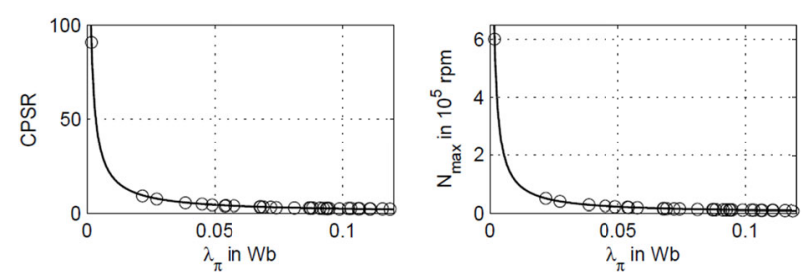

Fig. 3: Regression of CPSR and $N_{\max }$ as a function of $\sqrt{\lambda_{\pi}^{2}}$

\section{F. Conclusion}

All introduced RSM designs can adequately predict motor characteristics and parameters based on few advisedly chosen motor design samples. It became apparent that the choice of the regression model is only secondary as long as the significance of its shortcomings is considered and the input variables are selected wisely avoiding substantial nonlinearity. Sensitive parameters can be estimated using robust substitutes deduced from the analytic model. The preferential decision criterion should rather be the complexity of the subordinated experiments or computations. Since magnet and circuit properties can be easily changed using scripts, the determination of the response vector is automatable and suggests the use of highorder polynomials and the FFD. On the contrary, optimization problems considering geometrical motor parameters usual require manual modifications and are often initiated exerting first-order models which will be rearranged or improved in a second step. In this case flexible and efficient designs like the CCD are suited. It is the first choice for the optimization process in this paper as it provides the most precise regression results in the fringe areas of the exemplary experimental region. The BBD would be appropriate for an adjusted DoE as well as the FFD, if one can condone the high experimental costs.

\section{OPTIMIZATION}

In motor design naturally appear multi-objective optimization problems which lead to compromises, as the optimum designs for every objective are usually mutually exclusive. For instance high efficiency contradicts to low production costs or, like in this case, a high torque excludes a high maximum speed. There are various approaches to solve this problems, for instance evolution strategy methods (ES) [15] and in particular genetic algorithms (GA) [16]. They are already high developed and use genetic operators, like recombination and mutation, to manipulate prior solutions. Enhancements are then considered in further variations. They all tend to find Pareto optimal solution sets, means vectors of design variables $\mathrm{D}_{\gamma}$ whose variation does not decrease any response without increasing at least one another, while one is optimal regarding the others. The entirety of all vectors forms one or multiple so-called Pareto frontiers. Basically ES methods are controlled trial-and-error methods. The genetic operators minimize the number of 
trials by evaluation of every iterative step. Furthermore partial solutions and finally Pareto optimal solutions are connected to its causative machine design. But technically the optimal solution can be found without any knowledge about the underlying design parameters. For this purpose the most rudimentary of all statistical approaches is used, the Monte Carlo Method. Within a few seconds a large number of random vectors of design variables is generated and the responses are calculated. The visualization of the results reveals the Pareto frontier precisely.

\section{A. Pareto optimization with Monte Carlo Method}

Unavoidable improving one criterion adversely affects another. However a so-called Pareto optimal solution set of compromises can be found in between. For one preferable value of criterion A, the contrary criterion B is optimized. The entirety of all optimized combinations of A and B leads to the Pareto frontier, in case of two-dimensional problems a strictly monotonic function in the $\mathrm{AB}$ plane. For more than three objectives the visualization is hardly possible and the use of complex evaluation algorithms is inevitable. In general a multi-objective optimization problem can be formulated as:

$$
F(x)=\min \left(f_{1}(x), f_{2}(x), \ldots . f_{k}(x)\right)
$$

Wherein $\mathrm{f}$ are functions representing the $\mathrm{k}$ design objectives regarding the decision makers $\mathrm{x} 2 \mathrm{R}$, which are in this case the design variables. If an objective function is to be maximized, it needs to be transformed into its inverse monotonic function. The global optimum does not exist generally and is therefore called utopian solution.

$$
\begin{gathered}
x_{\text {utopian }} \in \mathcal{R} \text { if } \forall x \in \mathcal{R}, f_{i}\left(x_{\text {utopian }}\right) \leq f_{i}(x) \\
\text { for } i \in[1,2, \ldots k]
\end{gathered}
$$

A solution is Pareto dominant if

$$
\begin{gathered}
x_{\text {dominant }} \in \mathcal{R} \text { if } \\
f_{i}\left(x_{\text {dominant }}\right) \leq f_{i}(x) \text { for } i \in[1,2, \ldots k] \\
f_{j}\left(x_{\text {dominant }}\right) \leq f_{j}(x) \text { for } \exists i \in[1,2, \ldots k]
\end{gathered}
$$

And Pareto optimal, if it is the most dominant [17]

$$
\begin{gathered}
x^{P}=x_{\text {Pareto }} \in \mathcal{R} \text { if and only if there exist no } \\
f_{i}(x) \leq f_{i}\left(x_{\text {Pareto }}\right) \text { for } \forall i \in[1,2, \ldots k] \\
f_{j}(x) \leq f_{j}\left(x_{\text {pareto }}\right) \text { for } \exists i \in[1,2, \ldots k]
\end{gathered}
$$

The Pareto optimal design vectors $D^{P}$ are to be determined, which minimize the magnetic flux $\lambda_{d}$ at $\beta=\pi$ to zero:

$$
\min \left(f_{\lambda}\left(D_{\lambda}\right)\right)=0
$$

and maximize the maximum torque $\mathrm{T}_{\max }$, respectively minimize its negative $-\mathrm{T}_{\max }$ :

$$
\max \left(f_{T}\left(D_{\lambda}\right)\right) \Leftrightarrow \min \left(f_{-T}\left(D_{\lambda}\right)\right)
$$

Hence the two-objective optimization problem is:

$$
F\left(D_{\lambda}\right)=\min \left(f_{T}\left(D_{\lambda}\right), f_{-T}\left(D_{\lambda}\right)\right)
$$

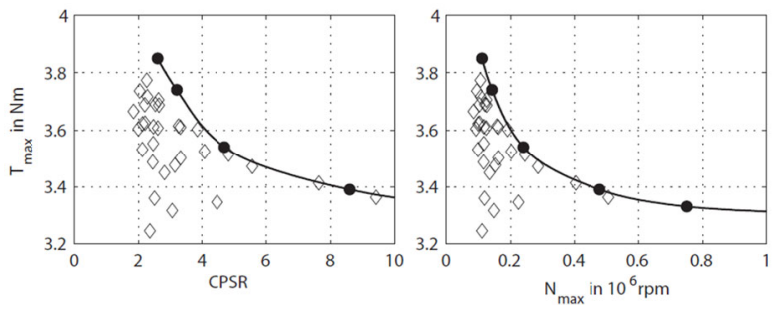

Fig. 4: Pareto optimal solutions of CPSR and $N_{\max }$ to $T_{\max }$.
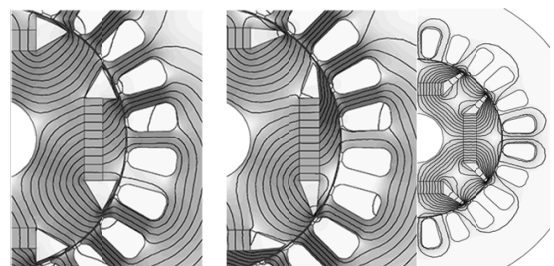

Fig. 5: Bending of flux lines in rotor path for different angles $\alpha_{2}$. (a) $x_{2}=$ min, maximum torque, (b) $x_{2}=1$, minimum torque, (c) almost total cancellation of air gap flux

And has the Pareto optimal solutions $\mathrm{D}_{\lambda}{ }^{\mathrm{P}}$, that means there exists no vector $D_{\lambda}$ with:

$$
f_{-T}\left(D_{\lambda}\right) \leq f_{-T}\left(D_{\lambda}^{P}\right) \cap f_{\lambda}\left(D_{\lambda}^{P}\right)<f_{\lambda}\left(D_{\lambda}^{P}\right)
$$

The functions $\mathrm{f}_{-\mathrm{T}}(\mathrm{D})$ and $\mathrm{f}_{\mathrm{T}}(\mathrm{D})$ are unknown. Indeed the second-order polynomials, provided by the RSM, can emulate them, but still an analytic optimization is not constructive. Rather the Pareto solutions are determined visually from the solutions attained by the Monte Carlo Method. The reading error is less than the regression error arising from the RSM. The particular design objective in this case is

$$
T_{\max }=f\left(\lambda_{\pi}\right)=f\left(\lambda_{m}, L_{d}, L_{q}, \beta_{T_{\max }}\right)=\max
$$

The Monte Carlo experiments are regressions results of the Response Surface Methodology and expected to be underneath:

$$
\hat{y}_{T_{\max }} \leq f\left(\hat{y}_{\lambda_{\pi}}\right)
$$

For a precise mapping of potential Pareto optimal solutions, up to 200000 random design vectors within $\mathfrak{R}$ are generated (Fig. 6). Using the polynomials provided by the CCD regression model, the predicted responses $y$ for $T_{\max }$ and $\lambda_{\pi}$ are determined in order to achieve a distinctive boundary. Fig. 5 a shows the predicted maximum for $\mathrm{T}_{\max }$, it can be seen that for the optimum design the air barriers prevent the flux lines to bend excessively within the rotor path (Fig 5b), force its bending into the air gap and therefore maximize the Maxwell forces. In contrary to the torque, the optimum field weakening characteristic $\lambda_{\pi}=0$ is clearly defined. It theoretically allows infinite speed and CPSR (Fig. 4), with a clear understanding that these speeds are mechanically impossible. Consequently the optimum field weakening design will seldom be applied practically as it decreases the torque for the whole speed range. Unless the maximum constant power output is desired up to the point of maximum speed. However motor designs which allow the complete cancellation (Fig. 5c) of the magnetic excitation field are theoretically interesting in terms of this paper as it 
illustrates the ability of the RSM to determine concrete objectives. So it will be shown that maximization problems are uncritical, since an exact value is irrelevant, as long as the maximum is distinctive. But a minimization to a certain value 'zero' requires several iterations and subsequently verifications of the predicted responses.

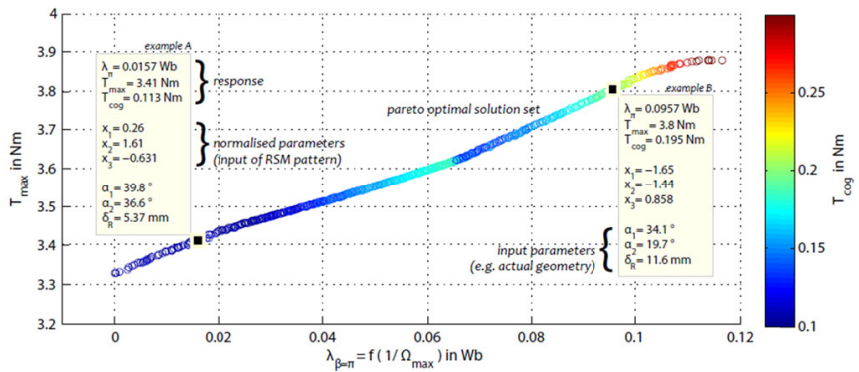

Fig. 6: Scatter plot of 200,000 responses, filtered for a twoobjective Pareto optimal solution set of maximum torque and field weakening ability (cancellation for $\lambda_{\pi}=0$ ) and adjusted for invalid parameter combinations; cogging torque as informative additional objective (color coded); immediate output of responses and their corresponding (normalized) design parameters; computation time less than 5 seconds on an average workstation assuming existing training data (by previous FE computations)

\section{CONCLUSION}

Traditional design procedures, based on analytic models and physical prototyping yield to satisfactory results for a wide range of motor types. Induction machines, synchronous motors with distributed windings and even salient pole SM and surface mounted PMSM can be modeled analytically accurate enough. The finite element analysis is then primarily used for the final optimization. However the negligence of saturation for IPMSM is not acceptable anymore and occasionally extraordinary shaped magnet circuits prohibit the use of analytical models and necessitate FE-analysis already during the design process. This is basically a time-consuming process as CAD drawings need to be created and modified and the computations are costly. With the Response Surface Methodology a procedure was introduced minimizing the effort to a few design samples. These, defined by a sophisticated design of experiments, are interpolated and therefore establish a consistent non-linear model of the machine taking into account all saturation effects. Motor parameters and performance characteristics can be estimated adequately for the entire space of design parameters. The determination of torque and power capability shows only minor regression errors of less than $2 \%$, while the field weakening ability is merely vaguely predictable as the influences of frequency dependent effects like iron losses and the skin effect of the windings are regarded inadequate. In case this is the main object of investigation, the model can be enhanced by including stator resistance, end-winding inductance and an iron loss factor. But even so the resisting torque due to friction constrains the maximum speed to less than a hundred thousand rpm, though the development of magnetic bearings obliterates this limitation.

\section{REFERENCES}

[1] K. Lewotsky. (2012, May) Dn insight: What rare earth shortages mean for engineers.www.designnews.com/author.asp? section_id=1386\&doc $\mathrm{id}=240050$

[2] K. Lewotsky. (2013) Market update: Rare earth magnet prices and motioncontrol.www.motioncontrolonline.org/i4a/pages/index.cfm?pageI $\mathrm{D}=4525$

[3] T. J. E. Miller, Brushless permanent-magnet and reluctance motor drives. Oxford: Clarendon Press ; New York : Oxford University Press, 1989.

[4] L. Jolly, "Design optimization of permanent magnet motors using response surface methodology and genetic algorithms," Master's thesis, National University of Singapore, 2005.

[5] T. Jahns, G. Kliman, and T. W. Nieumann, "Interior permanent-magnet synchronous motors for adjustable-speed drives," Industry Applications, IEEE Transactions on, vol. IA-22, no. 4, pp. 738-747, 1986.

[6] W. Soong and T. Miller, "Field-weakening performance of brushless synchronous ac motor drives," Electric Power Applications, IEE Proceedings, vol. 141, no. 6, pp. 331-340, 1994.

[7] S. Morimoto, Y. Takeda, T. Hirasa, and K. Taniguchi, "Expansion of

operating limits for permanent magnet motor by current vector control considering inverter capacity," Industry Applications, IEEE Transactions on, vol. 26, no. 5, pp. 866-871, 1990 .

[8] W. Soong and T. Miller, "Theoretical limitations to the field-weakening performance of the five classes of brushless synchronous ac motor drive," in Electrical Machines and Drives, 1993. Sixth International Conference on Conf. Publ. No. 376), 1993, pp. 127-132.

[9] R. Schiferl and T. Lipo, "Power icapability of salient pole permanent magnet synchronous motors in variable speed drive applications," Industry Applications, IEEE Transactions on, vol. 26, no. 1, pp. 115123,1990

[10] G. E. P. Box and K. B. Wilson, "On the experimental attainment of optimum conditions," Journal of the Royal Statistical Society. Series B (Methodological), vol. 13, no. 1, pp. 1-45, 1951.

[11] R. Rong, D. Lowther, Z. Malik, H. S., J. Nelder, and R. Spence, "Applying response surface methodology in the design and optimization of electromagnetic devices," Magnetics, IEEE Transactions on, vol. 33, no. 2, pp. 1916-1919, 1997.

[12] M. Jabbar, L. Q., and L. Jolly, "Application of response surface methodology (rsm) in design optimization of permanent magnet synchronous motors," in TENCON 2004 IEEE Region 10 Conference, vol. C, 2004, pp. 500-503 Vol. 3.

[13] A. I. Khuri and S. Mukhopadhyay, "Advanced review: Response Surface methodology," WIREs Computational Statistics, vol. 2, pp. 128$149,2010$.

[14] G. E. P. Box and D. W. Behnken, "Some new three level designs for the study of quantitative variables," Technometrics, vol. 2, no. 4, pp. 455-475, 1960.

[15] D. A. Van Veldhuizen and G. B. Lamont, "Multiobjective evolutionary algorithms: Analyzing the state-of-the-art," Evol. Computed, vol. 8, no. 2, pp. 125-147, 2000.

[16] K. Deb, A. Pratap, S. Agarwal, and T. Meyarivan, "A fast and elitist multiobjective genetic algorithm: Nsga-ii," Evolutionary Computation, IEEE Transactions on, vol. 6, no. 2, pp. 182-197, 2002.

[17] P. Ngatchou, A. Z., and M. El-Sharkawi, "Pareto multi objective optimization," in Intelligent Systems Application to Power Systems, 2005. Proceedings of the 13th International Conference on, 2005, pp. $84-91$.

\section{BIOGRAPHIES}

Robert Seifert was born in Freiberg, Germany in 1989. He is currently graduating in Electrical Engineering (M.Sc.) at the Department of Electrical Machines and Drives of the Dresden University of Technology. His main fields of interest are magnetic bearings, linear drives and feedback control systems.

Ramon Bargalló Perpiñà was born in Catalonia, Spain, 1962. He received the B.Sc., MSc, and $\mathrm{PhD}$ degrees in Industrial Engineering from the Polytechnic University of Catalonia (UPC), in 1985, 1995, and 2001 respectively. He is currently Professor in Electrical Engineering Department at EUETIB of UPC. Also he has about 50 papers on International Conferences and Journals. His employment experience includes assessment of some companies about the improvement of design of electrical machines using FE methodology. His research interest is in the field of design of electrical machines. 